\title{
Collectively Encoded Rydberg Qubit
}

\author{
Nicholas L. R. Spong $\odot,{ }^{1}$ Yuechun Jiao, ${ }^{1,2}$ Oliver D. W. Hughes, ${ }^{1}$ Kevin J. Weatherillø, ${ }^{1}$ \\ Igor Lesanovsky $\oplus^{3,4}$ and Charles S. Adams, \\ ${ }^{1}$ Department of Physics, Joint Quantum Centre Durham-Newcastle, \\ Rochester Building, Durham, England DH1 3LE, United Kingdom \\ ${ }^{2}$ State Key Laboratory of Quantum Optics and Quantum Optics Devices, Institute of Laser Spectroscopy, \\ Shanxi University, Taiyuan 030006, China \\ ${ }^{3}$ Institut für Theoretische Physik, Auf der Morgenstelle 14, 72076 Tübingen, Germany \\ ${ }^{4}$ School of Physics and Astronomy and Centre for the Mathematics and Theoretical Physics of Quantum Non-Equilibrium Systems, \\ The University of Nottingham, Nottingham, England NG7 2RD, United Kingdom
}

(Received 24 October 2020; accepted 28 June 2021; published 6 August 2021)

\begin{abstract}
We demonstrate a collectively encoded qubit based on a single Rydberg excitation stored in an ensemble of $N$ entangled atoms. Qubit rotations are performed by applying microwave fields that drive excitations between Rydberg states. Coherent readout is performed by mapping the excitation into a single photon. Ramsey interferometry is used to probe the coherence of the qubit, as well as to test the robustness to external perturbations. We show that qubit coherence is preserved even as we lose atoms from the polariton mode, preserving Ramsey fringe visibility. We show that dephasing due to electric field noise scales as the fourth power of field amplitude. These results show that robust quantum information processing can be achieved via collective encoding using Rydberg polaritons, and hence this system could provide an attractive alternative coding strategy for quantum computation and networking.
\end{abstract}

DOI: 10.1103/PhysRevLett.127.063604

Quantum technology is increasingly expanding our capabilities in computing, sensing, metrology, and communications. Atomic systems, including those exploiting highly excited Rydberg states, are particularly attractive for quantum applications [1-6] because they offer a unique combination of precision [7], high-fidelity entanglement generation [8-12], scaling to three dimensions [13,14], direct photonic readout $[15,16]$, and strong photon-photon interactions [17-21]. Recently, remarkable progress has been made using individual Rydberg atoms for quantum simulation [22-26]. In parallel and across the full spectrum of quantum computing platforms, there has been considerable recent interest in the use of collective encoding strategies exploiting different spatial modes [27-29], internal states [30,31], grid states [32,33], and Schrödinger cat states [34].

In this Letter, we demonstrate a new collective-coding scheme based on Rydberg polaritons [2,4,35]. The novel feature of our scheme is that the qubit is stored as a superposition of Rydberg polariton modes. One advantage of this scheme is that quantum information is distributed over many atoms as opposed to single atom encoding

Published by the American Physical Society under the terms of the Creative Commons Attribution 4.0 International license. Further distribution of this work must maintain attribution to the author(s) and the published article's title, journal citation, and DOI. schemes. An additional advantage is that the polariton phase [36] enables direct photonic state readout in a well-defined spatial mode [18]. Also, the collective character of both qubit states causes the Rabi frequency for qubit rotations to be independent of the number of atoms. Large transition dipole moments between highly excited Rydberg states (e.g., the radial matrix element for the $|r\rangle=\left|60 S_{1 / 2}\right\rangle$ to $\left|r^{\prime}\right\rangle=\left|60 P_{3 / 2}\right\rangle$ transition is $3684 \mathrm{D}$ [37]) provide for fast coherent control and excitation transfer [38] on timescales on the order of nanoseconds. Our scheme is scalable to many collective qubits using ensemble arrays [39], and it could provide an alternative hybrid strategy for quantum networking exploiting microwave interactions $[40,41]$.

The main focus of this Letter is to demonstrate coherent control of our collective qubit, as well as to test the robustness of the scheme to both atom loss and decoherence due to environmental noise [42]. The collective encoding scheme works as follows; see Fig. 1: For $N$ atoms within a blockade volume [35], the transition $|g\rangle \rightarrow$ $|e\rangle \rightarrow|r\rangle$ couples the $N$-atom ground state $|\mathrm{G}\rangle=$ $\left|g_{1}, g_{2}, \ldots, g_{j}, \ldots, g_{N}\right\rangle$ to the collective state

$$
|0\rangle=\frac{1}{\sqrt{N}} \sum_{j=1}^{N} e^{i\left(\boldsymbol{k} \cdot \boldsymbol{R}_{j}-\omega_{r} t\right)}\left|g_{1}, g_{2}, \ldots, r_{j}, \ldots, g_{N}\right\rangle,
$$

where $g_{j}$ and $r_{j}$ denote atom $j$, with position $R_{j}$ in states $|g\rangle$ and $|r\rangle$, respectively; see Fig. 1 . The phase at each atom 


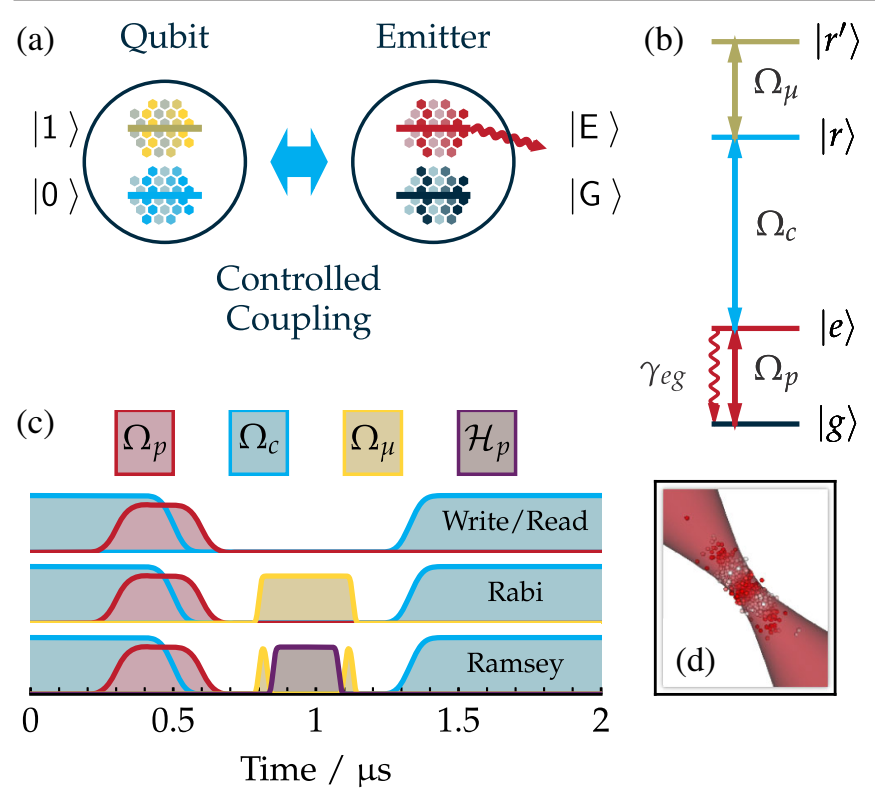

FIG. 1. Collective encoding and readout: (a) Quantum information encoded into a Rydberg polariton [36] in a superposition of $|0\rangle$ and $|1\rangle$, supported by Rydberg states $|r\rangle$ and $\left|r^{\prime}\right\rangle$; see Eqs. (1) and (2). Qubit coherently couples to a photon emitter, $|E\rangle$ and $|\mathrm{G}\rangle$ supported by $|e\rangle$ and $|g\rangle$. A control field (blue arrow) provides coupling with Rabi frequency $\Omega_{c}$. (b) Internal states of each atom. Initialization of qubit state $|0\rangle$ performed via twophoton excitation of transition $|g\rangle \rightarrow|e\rangle \rightarrow|r\rangle$. Single excitation of collective state $|0\rangle$ enforced by Rydberg blockade mechanism [35]. Qubit rotations implemented by driving transition $|r\rangle \leftrightarrow$ $\left|r^{\prime}\right\rangle$ using a microwave field with amplitude characterized by a Rabi frequency $\Omega_{\mu}$. Readout performed via polariton retrieval from $|r\rangle \rightarrow|e\rangle$, whereafter $|e\rangle$ decays back to ground state $|g\rangle$ with rate $\gamma_{e g}$ via collective emission into mode of original photon. (c) Pulse sequences used for qubit read-write, Rabi oscillations, and Ramsey interferometry. Perturbative Hamiltonians $\mathcal{H}_{p}$ (purple) can be implemented via external fields. (d) Illustration of our atomic ensemble in an optical tweezer.

contains both local phase terms $\mathbf{k} \cdot \mathbf{R}_{j}$, where $\mathbf{k}$ is the effective wave vector of the excitation lasers, and a global phase $-\omega_{r} t$, where $\omega_{r}$ is the angular frequency of the transition $|g\rangle \rightarrow|r\rangle$. For an ensemble initialized in $|0\rangle$, applying a microwave field with detuning $\Delta_{\mu}$ relative to the $|r\rangle \rightarrow\left|r^{\prime}\right\rangle$ transition [see Figs. 1(a) and 1(b)] couples $|0\rangle$ to the collective state

$$
|1\rangle=\frac{1}{\sqrt{N}} \sum_{j=1}^{N} e^{i\left(\boldsymbol{k} \cdot \boldsymbol{R}_{j}-\omega_{r^{\prime}} t\right)}\left|g_{1}, g_{2}, \ldots, r_{j}^{\prime}, \ldots, g_{N}\right\rangle
$$

Because both $|0\rangle$ and $|1\rangle$ contain $N$ terms, the Rabi frequency for qubit rotations is independent of the number of atoms $N$. This enables high-fidelity single-qubit rotations.

Finally, applying a coupling laser (blue in Fig. 1) resonant with the transition $|r\rangle \rightarrow|e\rangle$, we couple the collective state $|0\rangle$ to the state

$$
|\mathrm{E}\rangle=\frac{1}{\sqrt{N}} \sum_{j=1}^{N} e^{i \boldsymbol{k}^{\prime} \cdot \boldsymbol{R}_{j}}\left|g_{1}, g_{2}, \ldots, e_{j}, \ldots, g_{N}\right\rangle .
$$

This state decays on a timescale of $10 \mathrm{~ns}$ via collective emission of a single photon in a well-defined optical mode $[18,35]$. Measuring the occupation number of the optical mode performs a projective measurement of the qubit state.

The experimental sequence is illustrated in Fig. 1(c); see also Refs. [20,43-46]. The state $|0\rangle$ is initialized using a probe (red) and a coupling (blue) laser with Rabi frequencies $\Omega_{p}$ and $\Omega_{c}$ to drive the two-photon transition $|g\rangle \rightarrow|e\rangle \rightarrow|r\rangle$. Subsequently, we apply the microwave field, which is yellow in Fig. 1(c), with the Rabi frequency $\Omega_{\mu}$ to drive the qubit transition. Finally, the coupling laser is turned back on to perform the readout of state $|0\rangle$. The atomic ensemble of ${ }^{87} \mathrm{Rb}$ atoms is laser cooled and transferred to an optical tweezer trap with a wavelength of $862 \mathrm{~nm}$, a beam waist of $w_{0}=5 \mu \mathrm{m}$, and a trap depth of $\sim 0.5 \mathrm{mK}$. The ensemble is cooled to $50 \mu \mathrm{K}$ and optically pumped into the state $|g\rangle=\left|5 S_{1 / 2}, F=2, m_{F}=2\right\rangle$. We load a few thousand atoms prior to any loss due to photon scattering events in order to achieve the requisite $\mathrm{OD} \approx 4$ for photon storage and retrieval. Details on the preparation and optical response of our dipole traps can be found in Refs. [47,48].

The circularly polarized probe beam, generated by an external cavity diode laser, drives the

$$
\begin{aligned}
& |g\rangle=\left|5 S_{1 / 2}, F=2, m_{F}=2\right\rangle \rightarrow \\
& |e\rangle=\left|5 P_{3 / 2}, F^{\prime}=3, m_{F}^{\prime}=3\right\rangle
\end{aligned}
$$

transition on resonance. This light copropagates with the dipole trap and is focused to a $1 \mu \mathrm{m}$ beam waist at the center of the atomic ensemble. Probe pulses have a mean photon number of $\sim 0.25$ photons. This preserves optical depth (OD), and thus allows multiple experiments to be performed on the same ensemble. The coupling light, resonant with the $|e\rangle \rightarrow|r\rangle=\left|n S_{1 / 2}\right\rangle$ transition, is produced by a frequency-doubled diode laser system. The coupling beam is focused to $w_{0}=30 \mu \mathrm{m}$, and it counterpropagates with the probe. This coupling light is offset locked to a temperature stabilized optical cavity via electronic sidebands [49,50], and it can be tuned to address Rydberg states with principal quantum numbers of $n=30-95$. The blockade mechanism at high $n$ suppresses multiple Rydberg excitations such that the retrieved light is observed to have $g^{(2)}(t=0)=0.42 \pm 0.02$ for initialization and readout of the $|r\rangle=\left|60 S_{1 / 2}\right\rangle$. Single excitation purity can be enhanced by using higher lying Rydberg states to $g^{(2)}(t=0) \sim 0.15$ [44]. The efficiency of writing a polariton and retrieving a photon is between 0.5 and $1 \%$ for $n=60$. This limitation is imposed by motional dephasing, blockade, and finite ensemble optical depth. 
(a)
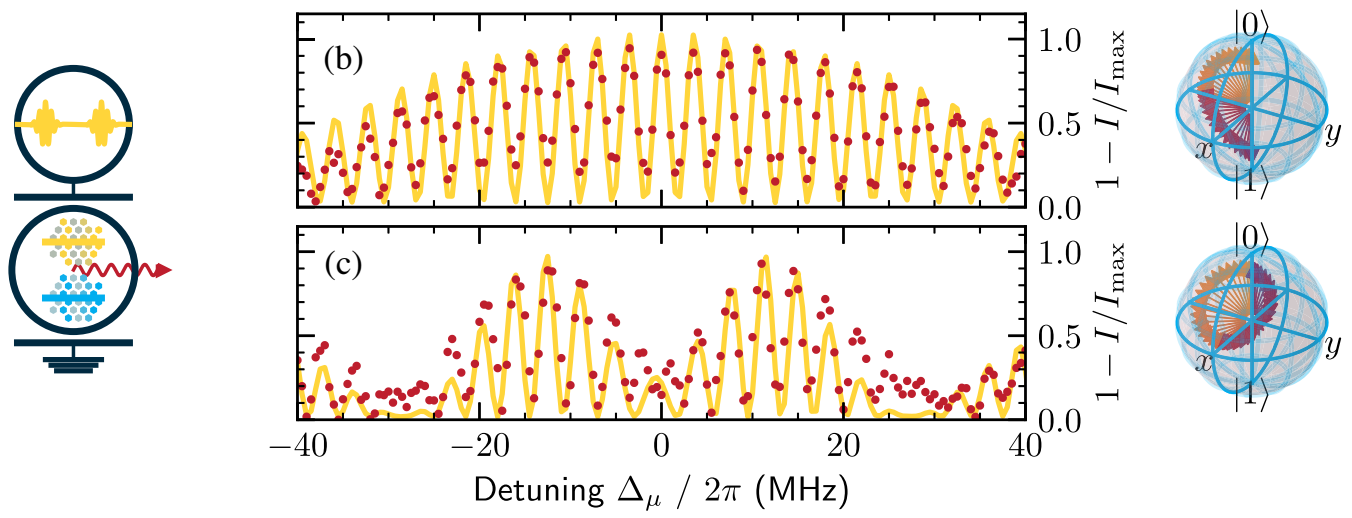

FIG. 2. Microwave manipulation of Rydberg qubits: Ramsey interferometry. (a) Qubit driven by two microwave pulses with duration of 30-50 ns separated by time $t_{\text {int }}=250 \mathrm{~ns}$. Polariton retrieval protocol converts population in $|0\rangle$ to photons (red arrow), which are counted. (b) Normalized photon counts $I / I_{\max }$ (red circles) as function of $\pi / 2$ pulse detuning $\Delta_{\mu}$ for case of $\Omega_{\mu} t_{\mu}=\pi / 2$ at $\Delta_{\mu}=0$. Monte Carlo simulation of data overlaid (yellow line). (c) Same as Fig. 2(b), except $\Omega_{\mu} t_{\mu}=\sqrt{2} \pi / 2$ such that, at $\left|\Delta_{\mu}\right|=\Omega_{\mu}=2 \pi$ (14 MHz); and we obtain a Hadamard gate. (d) Evolution on Bloch sphere for resonant Ramsey interferometry and double Hadamard operations.

Single-qubit rotations are driven by coupling the $|r\rangle=$ $\left|n S_{1 / 2}\right\rangle$ and $\left|r^{\prime}\right\rangle=\left|n^{\prime} P_{3 / 2}\right\rangle$ Rydberg states using a $16 \mathrm{~mm}$ in vacuo quarter-wave microwave antenna. The microwave source has a range of $0-40 \mathrm{GHz}$, driving single-qubit rotations for Rydberg qubits with $n>46$, with an $80 / 20 \%$ switching time of $10 \mathrm{~ns}$. Further experimental details can be found in Ref. [47]. All subsequent data in this Letter correspond to $|r\rangle=\left|60 S_{1 / 2}\right\rangle$ and $\left|r^{\prime}\right\rangle=\left|59 P_{3 / 2}\right\rangle$.

Figure 2 demonstrates coherent manipulation of a collective qubit. We observe quantum interference through Ramsey inteferometry using $\pi / 2$ pulses and Hadamard gates. The microwave pulse sequence is shown in Figs. 2(a) and 1(c) (bottom row). Two microwave pulses separated by $t_{\text {int }}=250$ ns perform single-qubit rotations in the $|0\rangle$ and $|1\rangle$ bases. The retrieved photon counts $I$ normalized to the maximum retrieved counts $I_{\max }$ as a function of the microwave detuning $\Delta_{\mu}$ for two values of the microwave pulse duration $t_{\mu}$ are shown in Figs. 2(b) and 2(c). In Fig. 2(b), the power $P$ and duration $t_{\mu}$ of each microwave pulse are chosen to give $\Omega_{\mu} t_{\mu}=\pi / 2$. In this case, the sequence of two $\pi / 2$ rotations about the $x$ axis in the Bloch sphere [see top of Fig. 2(d)] separated by a rotation about $z$ (free evolution) results in familiar Ramsey fringes. In Fig. 2(c), the pulse duration is increased to give $\Omega_{\mu} t_{\mu}=\sqrt{2} \pi / 2$. The special case of $\Delta_{\mu}=\Omega_{\mu}$ drives a Hadamard rotation ( $\pi$ rotation about a Bloch vector $45^{\circ}$ from the $z$ axis); see bottom of Fig. 2(d). Consequently, the maximum fringe visibility in Fig. 2(c) is observed at $\left|\Delta_{\mu}\right|=\Omega_{\mu}=2 \pi(12 \mathrm{MHz})$. The theoretical fits in Figs. 2(b) and 2(c) (yellow) are calculated by solving the two-level master equation for experimental parameters. We assume that the Rydberg state lifetime is long compared to the experimental timescale and that motional dephasing can be neglected due to postselection and normalization.
Next, to test the robustness of our collective encoding scheme, we apply a perturbation $\mathcal{H}_{p}$ during resonant Rabi oscillations or Ramsey interferometry; see Fig. 1(c). First, we explore a non-Hermitian perturbation, irreversibly removing atoms from the polariton by applying a scattering field (with the amplitude $\Omega_{s}$ resonant with $|g\rangle \rightarrow|e\rangle$ ) directed along the photon emission axis; see Fig. 3(a). Figures 3(b) and 3(c) illustrate the loss of visibility of Rabi oscillations and Ramsey fringes as a function of $\Omega_{s}$. The visibility $\mathbb{V}$ is defined as the difference between the peak and minimum signals normalized by their sum. Figure 3(d) shows the Ramsey fringes for low, intermediate, and high values of $\Omega_{s}$. Figure 3(e) shows the visibility of the Rabi oscillation and Ramsey fringes plus the normalized amplitude of the retrieved mode (pink triangles) labeled $\mathcal{F}$ for fidelity. In the Supplemental Material [51], the photon retrieval fidelity $\mathcal{F}$ is calculated from the Lindblad equation for a single stored Rydberg polariton driven by the scattering field for duration $t$,

$$
\mathcal{F}=\exp \left(-4 \frac{\Omega_{s}^{2}}{\gamma_{e g}} t\right)
$$

Here, $\gamma_{e g}$ is the lifetime of the state $|e\rangle$. The Rydberg state lifetimes $\tau_{r}$ and $\tau_{r^{\prime}}$ are assumed greater than the experimental timescale. Motional dephasing is omitted as experimental $\mathcal{F}$ are normalized to $\mathcal{F}\left(\Omega_{s}=0\right)$. The data are in good agreement with the model; see Fig. S1 in the Supplemental Material [51]. The exponential dependence apparent in Fig. 3(e) arises due to the averaging over many runs using the same ensemble.

The main result of Fig. 3(e) is that reducing the polariton retrieval amplitude (thus $\mathcal{F}$ ) by an order of magnitude only reduces the qubit coherence, which is characterized by the Ramsey fringe visibility, by a factor of two. Hence, we lose 

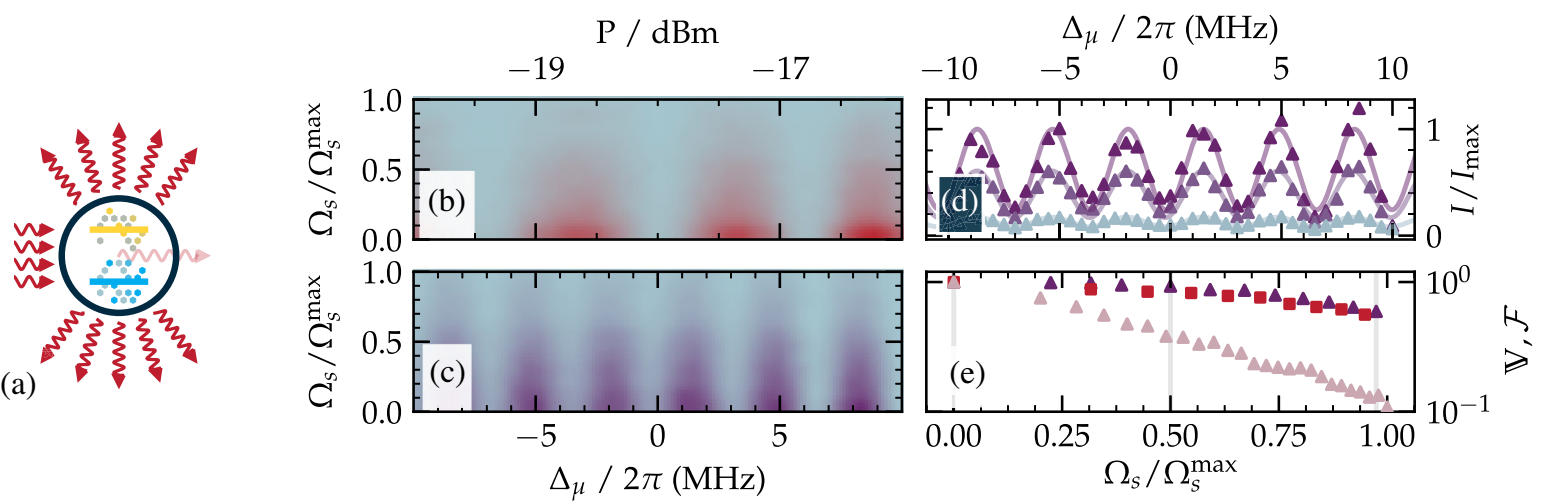

FIG. 3. Robustness of collectively encoded Rydberg qubit to non-Hermitian perturbation: (a) Scattering field with amplitude $\Omega_{s}$ applied with wave vector $\mathbf{k}_{s}$ along photon readout axis. (b) Rabi oscillation data: Heat map of normalized photon counts as function of microwave drive power $P$ and scattering field amplitude $\Omega_{s}$, up to $\Omega_{s}^{\max } / 2 \pi=1.6 \mathrm{MHz}$. Red and blue indicate high and low photon counts, respectively. (c) Ramsey fringes data: Heat map of normalized photon counts versus microwave detuning for increasing $\Omega_{s}$. Purple and blue indicate high and low photon counts, respectively. (d) Selected Ramsey fringe data at $\Omega_{s} / 2 \pi=0,1$, and $2 \mathrm{MHz}$ [see also vertical gray bars in Fig. 3(e)]. (e) Visibility $\mathbb{V}$ of Rabi oscillations (red squares) and Ramsey fringes (purple triangles) as a function of $\Omega_{s}$. Amplitude of polariton retrieval (readout fidelity; pink diamonds) degraded significantly faster than visibilities. Data in Figs. 3(b)-3(d) normalized to account for storage-retrieval efficiency $I_{\max } \mathbb{V}$ and $\mathcal{F}$ in Fig. 3(e) normalized to $\mathbb{V}_{0}$ and $\mathcal{F}_{0}$ (visibility and fidelity) at $\Omega_{s}=0$.

atoms from the polariton mode without significant degradation of the qubit coherence. In contrast to single-atom qubits where all the information is lost if a single atom is lost, our collective qubit is robust to atom loss.

Finally, we test the robustness of our collective encoding scheme against decoherence induced by environmental noise. Using a Tektronic AFG3252 arbitrary wave form generator with a bandwidth of $240 \mathrm{MHz}$, we apply an electrical noise pulse with peak-to-peak amplitude of $E_{\mathrm{Pk}-\mathrm{Pk}}=2 E_{0}$ to in vacuo electrodes; see Fig. 4(a). The noise pulse perturbs Rydberg energy levels via the Stark effect, and hence affects the global phase evolution of the collective states $|0\rangle$ and $|1\rangle$. This induces a $T 2$ type decay similar to the thermally induced decoherence in solid-state qubit systems [42]. The applied noise field can be modeled as a static field term, giving rise to a quadratic Stark shift, and a fluctuating field, causing a dephasing

$$
\mathcal{H}^{\prime}=\frac{1}{2}\left(\begin{array}{cc}
\alpha_{r^{\prime}} & 0 \\
0 & \alpha_{r}
\end{array}\right) \frac{E_{0}^{2}}{3}+\frac{1}{2}\left(\begin{array}{cc}
\alpha_{r^{\prime}} & 0 \\
0 & \alpha_{r}
\end{array}\right) \xi(t)
$$

where $\alpha_{j}$ is the polarizability of $|j\rangle$, and $\xi(t)$ represents the fluctuations of $E^{2}$ about the average value $E_{0}^{2} / 3$. Under the assumption of fast noise correlation decay of $\gamma_{\text {corr }}=1 / \tau_{\text {corr }}$ larger than other decay mechanisms, the dephasing rate

$$
\gamma_{\text {dep }}=\frac{4}{45} \cdot \frac{\left(\alpha_{r^{\prime}}-\alpha_{r}\right)^{2} E_{0}^{4}}{\hbar^{2}} \cdot \tau_{\text {corr }}
$$

where $\tau_{\text {corr }}$ is the noise correlation time; see Supplemental Material [51]. The Stark shift is observed experimentally and is apparent in Fig. 4(b). The predicted $E_{0}^{4}$ scaling is fit to the data in Fig. 4(c). In the Supplemental Material (see Fig. S2), we show that this quartic power law is a good fit. The data diverge from the quartic model at higher $E_{0}$,

(a)
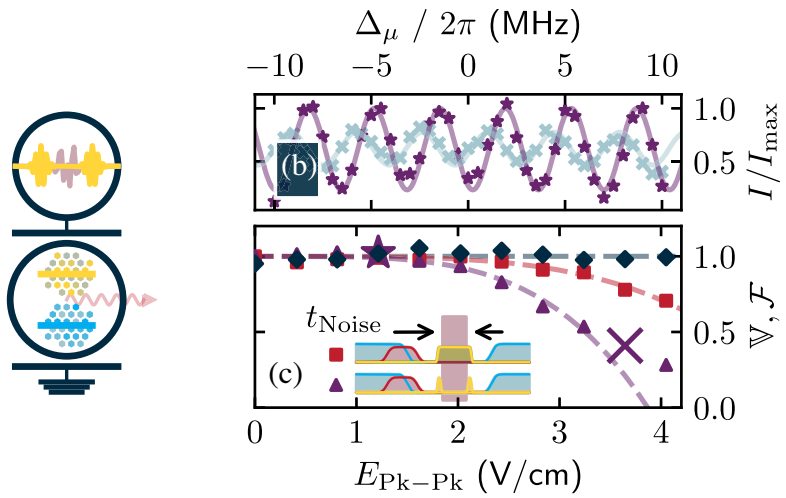

FIG. 4. Noise-induced dephasing: (a) Pulsed $E$ field with Gaussian amplitude noise applied during Ramsey and Rabi pulse sequences. Final state of qubit measured. (b) Ramsey fringes corresponding to central region of Fig. 2 for low noise $\left(E_{\mathrm{Pk}-\mathrm{Pk}}=1.2 \mathrm{~V} / \mathrm{cm}\right.$; purple stars $)$ and high noise $\left(E_{\mathrm{Pk}-\mathrm{Pk}}=3.6 \mathrm{~V} / \mathrm{cm}\right.$; blue crosses $)$. Solid lines are sinusoidal fits. (c) Main: Rabi oscillation (red squares) and Ramsey fringe (purple triangles) visibility as functions of noise amplitude. Applied noise does not affect fidelity (black diamonds). Both Rabi oscillation and Ramsey fringe visibility proportional to $E_{0}^{4}$ (red and purple dashed lines), as predicted by model. Ramsey visibility diverges from quartic model at large $E_{\mathrm{Pk}-\mathrm{Pk}}$ due to complex stark shifts. Star and cross show datasets detailed in Fig. 4(b). Inset of Fig. 4(c) shows duration of pulse, where $t_{\text {Noise }}$ is equal to total duration of Rabi or Ramsey sequence; see Fig. 1(c). $\mathbb{V}$ and $\mathcal{F}$ in Fig. 1(c) normalized to $\mathbb{V}_{0}$ and $\mathcal{F}_{0}$ (visibility and fidelity) at $E_{\mathrm{Pk}-\mathrm{Pk}}=0 \mathrm{~V} / \mathrm{cm}$. 
where the simplifying assumption of a quadratic Stark shift breaks down.

In summary, we propose and demonstrate a novel collective encoding scheme for qubits based on Rydberg polaritons. We demonstrate fast coherent control using microwave fields. We find Rydberg qubits to have excellent coherence properties, allowing for the implementation of fast Rabi oscillations, Ramsey interferometry, and Hadamard gates. By performing Ramsey interferometry, we demonstrate the robustness of a collectively encoded Rydberg qubit to depletion of atoms and to electric field noise. Rydberg qubits retain their quantum information even as the polariton suffers a partial loss of spatial phase coherence. We demonstrate that Rydberg qubit dephasing due to electrical noise depends quartically on the noise amplitude, which is in agreement with theoretical predictions. Enhanced resilience to electrical noise might be obtained by utilizing "magic" Rydberg states, where the polarizabilities of the Rydberg states are matched. Further work will focus on multiple qubits [44], qutrits, and phase gate proposals implemented using collective qubits [52,53].

This work was supported by Engineering and Physical Sciences Research Council Grants No. EP/M014398/1, No. EP/R002061/1, and No. EP/S015973/1. I. L. acknowledges support from the Deutsche Forschungsgemeinschaft through SPP 1929 (GiRyd) under Project No. 428276754, as well as from the Wissenschaftler-Rckkehrprogramm GSO/CZS of the Carl-Zeiss-Stiftung and the German Scholars Organization e.V.

*Corresponding author.

c.s.adams@durham.ac.uk

[1] M. Saffman, T. G. Walker, and K. Mølmer, Rev. Mod. Phys. 82, 2313 (2010).

[2] O. Firstenberg, C. S. Adams, and S. Hofferberth, J. Phys. B 49, 152003 (2016).

[3] M. Saffman, J. Phys. B 49, 202001 (2016).

[4] C. S. Adams, J. D. Pritchard, and J. P. Shaffer, J. Phys. B 53, 012002 (2020).

[5] M. Khazali and K. Mølmer, Phys. Rev. X 10, 021054 (2020).

[6] A. Browaeys and T. Lahaye, Nat. Phys. 16, 132 (2020).

[7] B. J. Bloom, T. L. Nicholson, J. R. Williams, S. L. Campbell, M. Bishof, X. Zhang, W. Zhang, S. L. Bromley, and J. Ye, Nature (London) 506, 71 (2014).

[8] Y.-Y. Jau, A. M. Hankin, T. Keating, I. H. Deutsch, and G. W. Biedermann, Nat. Phys. 12, 71 (2016).

[9] H. Levine, A. Keesling, A. Omran, H. Bernien, S. Schwartz, A. S. Zibrov, M. Endres, M. Greiner, V. Vuletić, and M. D. Lukin, Phys. Rev. Lett. 121, 123603 (2018).

[10] H. Levine, A. Keesling, G. Semeghini, A. Omran, T. T. Wang, S. Ebadi, H. Bernien, M. Greiner, V. Vuletić, H. Pichler, and M. D. Lukin, Phys. Rev. Lett. 123, 170503 (2019).
[11] T. M. Graham, M. Kwon, B. Grinkemeyer, Z. Marra, X. Jiang, M. T. Lichtman, Y. Sun, M. Ebert, and M. Saffman, Phys. Rev. Lett. 123, 230501 (2019).

[12] I. S. Madjarov, J. P. Covey, A. L. Shaw, J. Choi, A. Kale, A. Cooper, H. Pichler, V. Schkolnik, J. R. Williams, and M. Endres, Nat. Phys. 16, 857 (2020).

[13] M. Saffman and K. Mølmer, Phys. Rev. A 78, 012336 (2008).

[14] D. Barredo, V. Lienhard, S. de Léséleuc, T. Lahaye, and A. Browaeys, Nature (London) 561, 79 (2018).

[15] L. Li, Y. O. Dudin, and A. Kuzmich, Nature (London) 498, 466 (2013).

[16] J. Li, M.-T. Zhou, C.-W. Yang, P.-F. Sun, J.-L. Liu, X.-H. Bao, and J.-W. Pan, Phys. Rev. Lett. 123, 140504 (2019).

[17] J. D. Pritchard, D. Maxwell, A. Gauguet, K. J. Weatherill, M. P. A. Jones, and C. S. Adams, Phys. Rev. Lett. 105, 193603 (2010).

[18] Y. O. Dudin and A. Kuzmich, Science 336, 887 (2012).

[19] T. Peyronel, O. Firstenberg, Q.-Y. Liang, S. Hofferberth, A. V. Gorshkov, T. Pohl, M. D. Lukin, and V. Vuletić, Nature (London) 488, 57 (2012).

[20] D. Maxwell, D. J. Szwer, D. Paredes-Barato, H. Busche, J. D. Pritchard, A. Gauguet, K. J. Weatherill, M.P. A. Jones, and C.S. Adams, Phys. Rev. Lett. 110, 103001 (2013).

[21] D. Tiarks, S. Schmidt-Eberle, T. Stolz, G. Rempe, and S. Dürr, Nat. Phys. 15, 124 (2019).

[22] H. Bernien, S. Schwartz, A. Keesling, H. Levine, A. Omran, H. Pichler, S. Choi, A. S. Zibrov, M. Endres, M. Greiner, V. Vuletic, and M. D. Lukin, Nature (London) 551, 579 (2017).

[23] J. Zhang, G. Pagano, P. W. Hess, A. Kyprianidis, P. Becker, H. Kaplan, A. V. Gorshkov, Z. X. Gong, and C. Monroe, Nature (London) 551, 601 (2017).

[24] S. De Léséleuc, V. Lienhard, P. Scholl, D. Barredo, S. Weber, N. Lang, H. P. Büchler, T. Lahaye, and A. Browaeys, Science 365, 775 (2019).

[25] P. Scholl, M. Schuler, H. J. Williams, A. A. Eberharter, D. Barredo, K.-N. Schymik, V. Lienhard, L.-P. Henry, T. C. Lang, T. Lahaye, A. M. Läuchli, and A. Browaeys, arXiv:2012.12268.

[26] S. Ebadi, T. T. Wang, H. Levine, A. Keesling, G. Semeghini, A. Omran, D. Bluvstein, R. Samajdar, H. Pichler, W. W. Ho, S. Choi, S. Sachdev, M. Greiner, V. Vuletic, and M. D. Lukin, arXiv:2012.12281.

[27] K. Tordrup, A. Negretti, and K. Mølmer, Phys. Rev. Lett. 101, 040501 (2008).

[28] C. Grezes, B. Julsgaard, Y. Kubo, M. Stern, T. Umeda, J. Isoya, H. Sumiya, H. Abe, S. Onoda, T. Ohshima, V. Jacques, J. Esteve, D. Vion, D. Esteve, K. Mølmer, and P. Bertet, Phys. Rev. X 4, 021049 (2014).

[29] V. Ranjan, J. O'Sullivan, E. Albertinale, B. Albanese, T. Chanelière, T. Schenkel, D. Vion, D. Esteve, E. Flurin, J. J. L. Morton, and P. Bertet, Phys. Rev. Lett. 125, 210505 (2020).

[30] E. Brion, K. Mølmer, and M. Saffman, Phys. Rev. Lett. 99, 260501 (2007).

[31] E. Brion, L. H. Pedersen, M. Saffman, and K. Mølmer, Phys. Rev. Lett. 100, 110506 (2008). 
[32] C. Flühmann, T. L. Nguyen, M. Marinelli, V. Negnevitsky, K. Mehta, and J. P. Home, Nature (London) 566, 513 (2019).

[33] P. Campagne-Ibarcq, A. Eickbusch, S. Touzard, E. Zalys-Geller, N. E. Frattini, V. V. Sivak, P. Reinhold, S. Puri, S. Shankar, R. J. Schoelkopf, L. Frunzio, M. Mirrahimi, and M. H. Devoret, Nature (London) 584, 368 (2020).

[34] A. Grimm, N. E. Frattini, S. Puri, S. O. Mundhada, S. Touzard, M. Mirrahimi, S. M. Girvin, S. Shankar, and M. H. Devoret, Nature (London) 584, 205 (2020).

[35] M. D. Lukin, M. Fleischhauer, R. Cote, L. M. Duan, D. Jaksch, J. I. Cirac, and P. Zoller, Phys. Rev. Lett. 87, 037901 (2001).

[36] M. Fleischhauer and M. D. Lukin, Phys. Rev. Lett. 84, 5094 (2000).

[37] N. Šibalić, J. Pritchard, C. Adams, and K. Weatherill, Comput. Phys. Commun. 220, 319 (2017).

[38] D. Barredo, H. Labuhn, S. Ravets, T. Lahaye, A. Browaeys, and C. S. Adams, Phys. Rev. Lett. 114, 113002 (2015).

[39] Y. Wang, S. Shevate, T. M. Wintermantel, M. Morgado, G. Lochead, and S. Whitlock, npj Quantum Inf. 6, 54 (2020).

[40] H. J. Kimble, Nature (London) 453, 1023 (2008).

[41] T. van Leent, M. Bock, R. Garthoff, K. Redeker, W. Zhang, T. Bauer, W. Rosenfeld, C. Becher, and H. Weinfurter, Phys. Rev. Lett. 124, 010510 (2020).

[42] A. Chenu, M. Beau, J. Cao, and A. del Campo, Phys. Rev. Lett. 118, 140403 (2017).
[43] J. D. Pritchard, Cooperative Optical Non-Linearity in a Blockaded Rydberg Ensemble, Springer Theses (Springer, Berlin, 2012), p. 210.

[44] H. Busche, P. Huillery, S. W. Ball, T. Ilieva, M. P. A. Jones, and C.S. Adams, Nat. Phys. 13, 655 (2017).

[45] C. Möhl, N. L. R. Spong, Y. Jiao, C. So, T. Ilieva, M. Weidemüller, and C.S. Adams, J. Phys. B 53, 084005 (2020).

[46] Y. Jiao, N. L. R. Spong, O. D. W. Hughes, C. So, T. Ilieva, K. J. Weatherill, and C. S. Adams, Opt. Lett. 45, 5888 (2020).

[47] H. Busche, S. W. Ball, and P. Huillery, Eur. Phys. J. Special Topics 225, 2839 (2016).

[48] R. J. Bettles, T. Ilieva, H. Busche, P. Huillery, S. W. Ball, N. L. R. Spong, and C. S. Adams, arXiv:1808.08415.

[49] J. I. Thorpe, K. Numata, and J. Livas, Opt. Express 16, 15980 (2008).

[50] R. Legaie, C. J. Picken, and J. D. Pritchard, J. Opt. Soc. Am. B 35, 892 (2018).

[51] See Supplemental Material at http://link.aps.org/ supplemental/10.1103/PhysRevLett.127.063604 for derivations of equations for fidelity and noise sensitivity found in this letter, as well as further information on the acquisition and analysis of experimental data.

[52] D. Paredes-Barato and C. S. Adams, Phys. Rev. Lett. 112, 040501 (2014).

[53] M. Khazali, C. R. Murray, and T. Pohl, Phys. Rev. Lett. 123, 113605 (2019). 